INPLASY

PROTOCOL

To cite: Huang et al.

Conservative versus conventional oxygen therapy for critically ill patients: a Metaanalysis of randomized controlled trials. Inplasy protocol 202070044. doi: 10.37766/inplasy2020.7.0044

Received: 12 July 2020

Published: 12 July 2020

Corresponding author: Hui-Bin Huang

hhba02922@btch.edu.cn

Author Affiliation:

Beijing Tsinghua Changgung Hospital

Support: None.

Review Stage at time of this submission: Preliminary searches.

Conflicts of interest:

The authors declare that they have no competing interests.

\section{Conservative versus conventional oxygen therapy for critically ill patients: a Meta-analysis of randomized controlled trials}

Huang, HB1; Chen, XL2; Zhang, BL3; Lin, $\mathrm{H}^{4}$.

Review question / Objective: We aim to perform a systemic review and meta-analysis to determine if conservative oxygenation versus conventional oxygenation results in a statistically significant difference in outcomes in critically ill adult patients.

Condition being studied: Authors of the current study come from a tertiary hospital in China and all the members have extensive experience in treating septic shock with ECMO. Furthermore, these authors have published several metaanalyses, which can guarantee the completion of the current study.

Information sources: We will search the references in the included studies and personal files. We will request advice from experts in the field. Additionally, we will search associated articles from critical care, surgical, infection meetings; and contacted the authors of included trials, if need.

INPLASY registration number: This protocol was registered with the International Platform of Registered Systematic Review and Meta-Analysis Protocols (INPLASY) on 12 July 2020 and was last updated on 12 July 2020 (registration number INPLASY202070044).

\section{INTRODUCTION}

Review question / Objective: We aim to perform a systemic review and metaanalysis to determine if conservative oxygenation versus conventional oxygenation results in a statistically significant difference in outcomes in critically ill adult patients.
Condition being studied: Authors of the current study come from a tertiary hospital in China and all the members have extensive experience in treating septic shock with ECMO. Furthermore, these authors have published several metaanalyses, which can guarantee the completion of the current study. 


\section{METHODS}

Participant or population: Adult ( $\geq 18$ years old) ICU patients.

Intervention: Adult critically ill patients receiving conservative oxygen therapy during their stay in ICU.

Comparator: Adult critically ill patients receiving conventional oxygen therapy during their stay in ICU.

Study designs to be included: We will include only randomised controlled trials in our study.

Eligibility criteria: We will include RCTs focusing adults ( $\geq 18$ yr or older) critically ill patients receiving conservative oxygen therapy verus. conventional oxygen therapy.

Information sources: We will search the references in the included studies and personal files. We will request advice from experts in the field. Additionally, we will search associated articles from critical care, surgical, infection meetings; and contacted the authors of included trials, if need.

Main outcome(s): Primary outcome is longest follow-up mortality. Secondary outcomes include oxygen exposures (i.e., SpO2, $\mathrm{PaO} 2$ and $\mathrm{PaO2:FiO2)}$ after treatment; mechanical ventilation free days; the length of stay in the ICU and hospital; $\triangle$ SOFA score and adverse events (as reported by authors).

Quality assessment / Risk of bias analysis: The Cochrane risk of bias tool will be adopted to assess the risk of bias for each RCT. For each RCT, risk of bias is evaluated as following: 1) random sequence generation; 2) allocation concealment; blinding of participants and personnel; 3) blinding of outcome assessment; 4) incomplete outcome data; 5) selective reporting; 6) other bias. Meanwhile, we also perform estimation on "overall" risk of bias. For each domain, risk of bias was categorized as "low," "unclear," or "high."
Disagreement for all methodological steps will be resolved by discussion.

Strategy of data synthesis: An overall effect estimate for all data as risk ratio (RR) / mean difference (MD) with $95 \% \mathrm{Cl}$ will be calculated. The presence of statistical heterogeneity among the studies by using the $Q$ statistics and the heterogeneity by using the 12 statistic was addressed. A p value of less than 0.10 or an 12 value of greater than $50 \%$ as indicative was considered of substantial heterogeneity. A random-effects model or a fixed-effects mode (DerSimonian-Laird) will be chosen when significant heterogeneity or nonsignificant heterogeneity was not observed, respectively.

Subgroup analysis: We will perform subgroup analyses of all causes of mortality rates according to the included studies with or without severe acute respiratory failure patients included.

Sensibility analysis: None.

Language: No language limitation was imposed.

Country(ies) involved: China.

Keywords: Conservative oxygen therapy; conventional oxygen therapy; critically ill; meta-analysis.

Contributions of each author:

Author 1 - Hui-Bin Huang - H-BH will be responsible for the contribution of conception, design, data interpretation, manuscript revision for critical intellectual content, and supervision of the study.

Author 2 - Xiao-Li Chen - X-LC will be responsible for the search of the scientific literature and drafted the manuscript.

Author 3 - Bei-Lei Zhang - B-LZ will be responsible for contribution of conception, design and data interpretation.

Author 4 - Hui Lin - HL will be responsible for helping to collect the data and performed statistical analyses. 